\title{
Dynamics of transfer of electron excitation in a donor-acceptor system with a carbon chain and ways of its relaxation
}

\author{
M.M. Sevryukova, Yu.P. Piryatinsky \\ Institute of Physics, National Academy of Sciences of Ukraine \\ 46, prospect Nauky, 03680 Kyiv, Ukraine \\ E-mail: yupiryat@iop.kiev.ua
}

\begin{abstract}
The optical properties and dynamics of transport of electron excitation and the ways of its relaxation in the supramolecular $\mathrm{D}-\pi-\mathrm{A}$ complex on the basis of merocyanines have been investigated. There have been found two components in the transfer of charge: fast and slow, which correspond to different conformational states of the carbon chain in merocyanines. It was found that the main photoluminescence of the studied molecular solutions of merocyanines by its nature is similar to the exciplex luminescence, as a manifestation of resonant and charge transfer interaction in an excited state. The lifetime in this state is about 2000 ps.
\end{abstract}

Keywords: merocyanines, photoluminescence, time-resolved emission spectra, charge transfer, donor-acceptor system, carbon chain.

Manuscript received 04.10.17; revised version received 20.11.17; accepted for publication 07.12.17; published online 07.12.17.

\section{Introduction}

The investigated $\mathrm{D}-\pi-\mathrm{A}$ compounds with intramolecular charge transport contain donor (D) and acceptor (A) molecular fragments interconnected by a conjugated $\pi$ electron system of methine groups. A feature of these supramolecular $\mathrm{D}-\pi-\mathrm{A}$ complexes is the optically stimulated intramolecular transfer of electronic density between the $\mathrm{D}$ and $\mathrm{A}$ fragments through a conductive $\pi$ conjugated carbon chain. The conformational changes observed in the optical excitation of $\mathrm{D}-\pi-\mathrm{A}$ complexes can significantly effect on redistribution of electronic density in the carbon chain and on its transport properties. Theoretically, the process of electron transport in molecular $\mathrm{D}-\pi-\mathrm{A}$ complexes has been considered within the framework of the one-dimensional model in the work [1].

The environment effects on the electronic properties of the investigated $\mathrm{D}-\pi-\mathrm{A}$ complexes significantly. The transfer of charge in the $\mathrm{D}-\pi-\mathrm{A}$ complexes during optical excitation depends on polarity of the solvent and its temperature. Due to the strong polarizability of $\pi$-electrons weakly connected with the nuclear core [2], the $\mathrm{D}-\pi-\mathrm{A}$ complexes are capable to reveal significant nonlinear optical activity. This is due to the considerable interest in these substances in the field of modeling the molecular structures with given electronic properties, studying their sensor and nonlinear optical properties, searching for methods to predict the values of their hyperpolarizability, using these substances as sensors.

In this paper, using as $\mathrm{D}-\pi-\mathrm{A}$ complexes, we have investigated merocyanines containing nitrogen heterocycles as D fragments and the malonodinitrile residue as A finite groups, and which are interconnected by a chain of methine groups. The molecules of the studied merocyanines have a relatively simple chemical structure that can be easily modified in order to obtain both linear and nonlinear characteristics. Despite the considerable number of studies of the merocyanine $\mathrm{D}-\mathrm{A}$ complexes by this time, there are many questions about the nature of excited states. The spectral properties of 
merocyanines in the excited state are close to the properties of symmetric cyanines [3-6], where the socalled "cyanine limit" states can be realized, that is, a state with the maximally aligned bond lengths is achieved. In optical excitation of the molecular solutions of merocyanines, the conformational state of the entire fluorophore, the polarity of the solvent and its temperature have a significant influence on the intramolecular charge transfer and its dynamics.

Using the low-temperature, time-resolved picosecond fluorescence spectroscopy, we have investigated the dynamics of the electronic properties of the merocyanine molecules in the excited state and the effect of the conformational state of fluorophore on the mobility of the electron density wave along it.

\section{Experimental part. Research substances and methods of experiment}

The optical properties and dynamics of the electron excitation transfer in the D- $\pi-\mathrm{A}$ complexes based on the M-2 merocyanine (2-\{5,5-dimethyl-3-[2-(2,3,6,7-tetrahydro-1 $H, 5 H$-pyrido[3,2,1-ij]quinolin-9-yl)vinyl]cyclohex-2-en-1-ylidene malononitrile) were investigated, its structural formula is shown in Fig. 1. The procedure for the synthesis of the compound was described in the papers $[7,8]$. In the molecule, two trimethylene bridges are used to block the conformational changes of the amino group. Introduction of methylene bridges into the chain provides a relatively high stability of substances.

Absorption spectra were measured using the Lambda 1050UV/VIS/NIR (Perkin Elmer) spectrometer. The stationary photoluminescence (PL) spectra were measured using the USB2000 + UV-VIS-ES spectrometer through an optical fiber with the diameter of $600 \mu \mathrm{m}$. To excite stationary PL, LEDs LLS-270, 385, 390 (Ocean Optics B.V.) were used. To excite nonstationary $\mathrm{PL}$ in the visible spectral region, a semiconductor laser EPL-405 with the wavelength $\lambda_{e}=$ $405 \mathrm{~nm}$ and the pulse duration $40 \mathrm{ps}$ was used. The frequency of bandpass of emission pulses can be regulated within the range $20 \mathrm{MHz}-10 \mathrm{kHz}$. To excite $\mathrm{PL}$ in the UV region, a pulsed LED with $\lambda_{e x}=255 \mathrm{~nm}$ and pulse duration 900 ps was used.

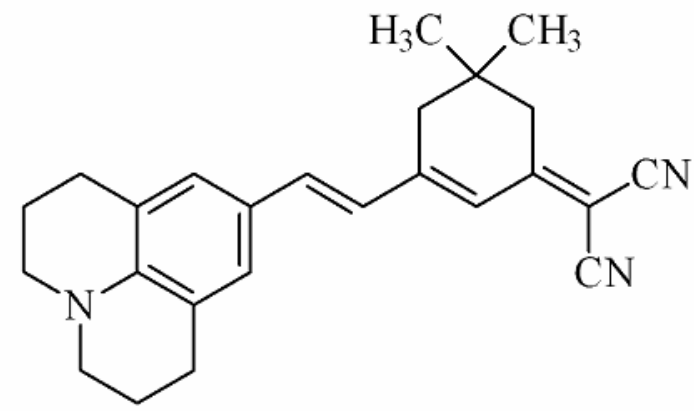

Fig. 1. Structural formula of the studied M-2 merocyanine.
To determine the lifetime $\tau$ of excited states of molecules, the technique of time-correlated single photon counting with picosecond resolution in time was used. This method is often used to measure the kinetics of PL decay of weakly emitting specimens at characteristic lifetimes $10^{-6} \ldots 10^{-11} \mathrm{~s}$. To excite PL, in this method a sequence of short excitation pulses of emission from semiconductor lasers with a strictly fixed consecution period is used. The probability of detecting PL is maintained at a level lower than one photon, when the object is excited by one pulse, and the repetition rate of the excitation pulse sets as high as possible. On the other hand, the pulse sequence is maintained such that the time interval between the pulses is at least 5 to 10 times longer than the time of PL decay that is measured. The resulting time dependence of PL was approximated by the expression:

$$
I(t)=I R F \sum_{i=1}^{n} A_{i} \exp \left(-\frac{t}{\tau_{i}}\right)
$$

where IRF (Instrument Response Function) is the function of the response of the detector to the laser pulse, $i$ - serial number, $\tau$ - lifetime of the excited state, $A_{i}$ - weight coefficient.

To measure the response function $I R F$, a certified colloidal solution supplied with the device was used. $I R F$ was taken into account when measuring the PL lifetimes close to the duration of the laser pulse.

To establish the true curve of PL attenuation according to experimental data, it is necessary to solve the integral equation

$$
I(t)=\int_{0}^{t} F\left(t-t^{\prime}\right) G\left(t^{\prime}\right) d t^{\prime}
$$

where $I(t)$ is the experimental dependence of the PL intensity on time, $F(t)$ - true dependence of the PL decay on time, $G(t)$ - Instrument Response Function $(I R F)$. To calculate $\tau$ and plot the time-resolved emission spectra (TRES) in the measuring system, there is a necessary software package. Using the sequence of kinetic curves of PL for different wavelengths of emission of molecular solution with merocyanines, it was possible to plot the spectral dependence of their PL on the delay time $t_{d}$ after peaking the exciting laser pulse - the spectra resolved in the picosecond time range. Experiments were carried out using the LifeSpec II spectrofluorometer (Edinburgh Instruments Ltd, UK) measuring system.

For excitation of two-photon PL, the base radiation of the Nd:YAG laser was used ( $\lambda_{e x}=1064 \mathrm{~nm}, t=10 \mathrm{~ns}$, $N=0.05-1.0 \mathrm{MW}, f=50 \mathrm{~Hz}$ ), and for the one-photon $\mathrm{PL}$ - the second harmonic of the Nd:YAG laser $\left(\lambda_{e x}=532 \mathrm{~nm}, N=0.1-0.5 \mathrm{MW}, f=50 \mathrm{~Hz}\right)$. The registration of one-photon PL was carried out by using a stroboscopic system $[21,22]$ that allowed recording the 
kinetics and PL spectra with a different time delay $t_{d}$ in relation to the laser pulse. As a spectral device, a monochromator MDR-12 was used. Registration of the spectra of one- and two-photon-excited PL was carried out at the maximum of laser pulse with a time window of 100 ps.

\section{Results of the experiment}

\subsection{Absorption spectra and stationary PL spectra of molecular solutions}

Fig. 2 shows the absorption spectra of the molecular solutions (with the concentration $c=2 \cdot 10^{-6} \mathrm{~mol} / \mathrm{l}$ ) in weakly polar toluene (curve 1 ) and polar acetonitrile (curve 2) at $300 \mathrm{~K}$. Broad spectral bands in the visible spectral region correspond to the first singlet $\left(\mathrm{S}_{0} \rightarrow \mathrm{S}_{1}\right)$ $\pi \pi^{*}$-electronic transition in the molecules under study. The position of the peaks of bands of absorption spectra for molecular solutions in the spectral range of $400 \ldots 800 \mathrm{~nm}$ (Fig. 2) is almost independent of the polarity of the solvent. An increase in the polarity of the solvent and a decrease in temperature resulted only in the widening of the absorption spectra bands from the long-wave side.

Fig. 3 shows absorption spectra (curve 1) and PL (curve 2) of molecular solutions in acetonitrile at room temperature in the spectral region of $250 \ldots 800 \mathrm{~nm}$. The absorption bands with the maxima at 278,345 , and $545 \mathrm{~nm}$ are typical for the solution. The bands with the maxima at 350 and $706 \mathrm{~nm}$ can be identified in the PL spectra. Fig. 3 shows that the PL spectra of molecular solutions in acetonitrile at room temperature have a significant long-wave shift respecting to absorption spectra. The Stokes shift at room temperature is $161 \mathrm{~nm}$ $\left(4190 \mathrm{~cm}^{-1}\right)$.

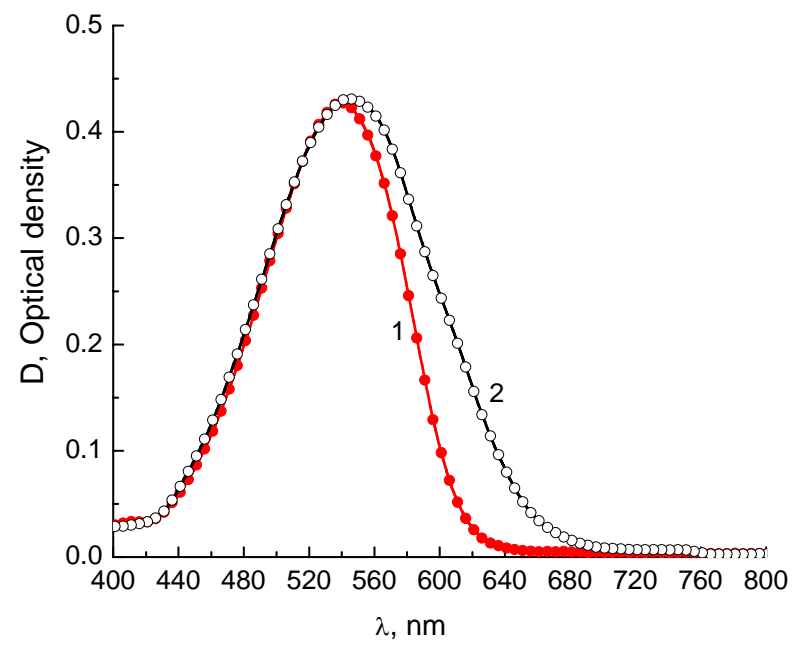

Fig. 2. Absorption spectra of $\mathrm{M}-2$ molecular solutions in toluene (1) and acetonitrile (2) at $300 \mathrm{~K}$.

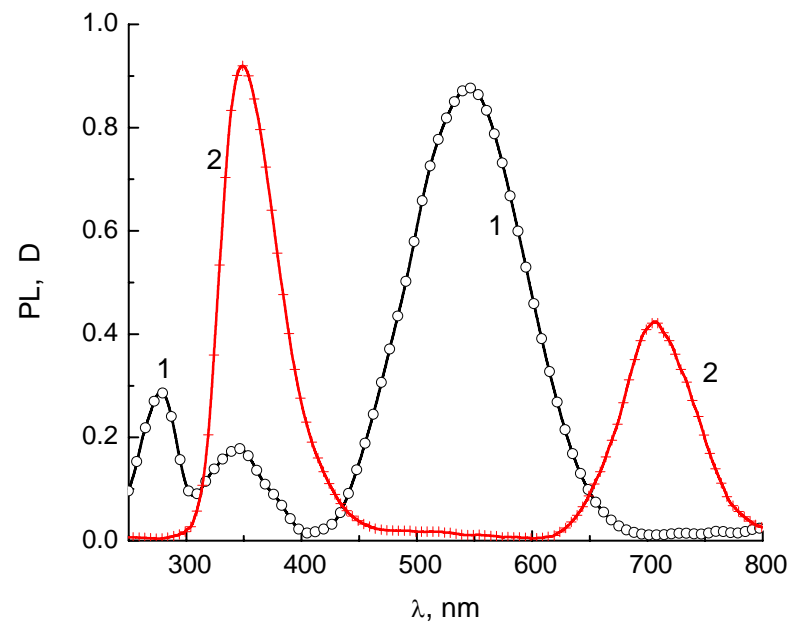

Fig. 3. Absorption spectra (1) and PL (2) of molecular solutions M-2 in acetonitrile at $300 \mathrm{~K}, \lambda_{e x}(\mathrm{PL})=255 \mathrm{~nm}$.

The dependence of the PL spectra of M-2 molecular solutions at room temperature on the wavelength $\lambda_{e x}$ of the exciting light was also investigated. The PL spectra at room temperature did not change with a change of $\lambda_{e x}$ from 250 to $670 \mathrm{~nm}$. For the PL spectra in the long-wave side from $740 \mathrm{~nm}$, a weak shoulder was observed. For the PL spectra of M-2 molecular solutions, a weak shoulder was observed in a more long-wave range than $740 \mathrm{~nm}$, which correlates with appearance of an inhomogeneous extension in absorption spectra in a polar solvent (Fig. 2).

Fig. 4a shows the stationary PL spectra of molecular solutions of merocyanine in slightly polar toluene at $4.2 \mathrm{~K}$ (curve 1 ) and $296 \mathrm{~K}$ (curve 2), when exciting with a nitrogen laser at the wavelength $\lambda_{e x}=$ $337.1 \mathrm{~nm}$. It should be noted that the quantum yields of PL of the studied solutions at room temperature are significantly lower than those at low temperatures. The structure of the spectra and positions of the maxima of PL bands of molecular solutions in toluene does not significantly depend on the change in temperature from room to helium. In the PL spectrum at $4.2 \mathrm{~K}$, the band with the maximum of $625 \mathrm{~nm}$ and long-wave shoulder at $680 \mathrm{~nm}$ can be distinguished.

In contrast to the absorption spectra that are weakly dependent on the solvent polarity, we observed significant differences in the PL spectra at room temperature when the solvent polarity was changed (Fig. 4b). These differences are related with significant conformational changes in the carbon chain and dipole moment of the merocyanine molecules in the excited state in a polar solvent. It is known from literature sources that, for many solutions of dyes at low temperatures, the PL spectra should not depend on the polarity of the solvent, since at low temperatures solvation processes are practically absent. In fact, as can be seen from Fig. 4b, differences in the PL spectra of acetonitrile solutions of the investigated dyes are 
observed, when the temperature changes from 296 to $4.2 \mathrm{~K}$. It is obvious that, in the ground state, some part of the molecules of merocyanine in the solution under cooling undergoes conformational changes. This is manifested as the long-wave expansion of absorption spectra of molecular solutions in a polar solvent.

The PL spectra of investigated solutions in acetonitrile, in contrast to the PL spectra of these molecular solutions in slightly polar toluene, depend significantly on temperature (Fig. 4b). When the temperature decreases from 296 down to $4.2 \mathrm{~K}$, the PL spectra were substantially displaced to the short-wave spectral region. For the acetonitrile solution at room temperature in the PL spectrum, the band with the maximum $706 \mathrm{~nm}$ is revealed (Fig. $4 \mathrm{~b}$, curve 2). When the temperature drops to $4.2 \mathrm{~K}$, there is a band with a maximum of $660 \mathrm{~nm}$ in the PL spectrum (Fig. 4b, curve 1$)$.

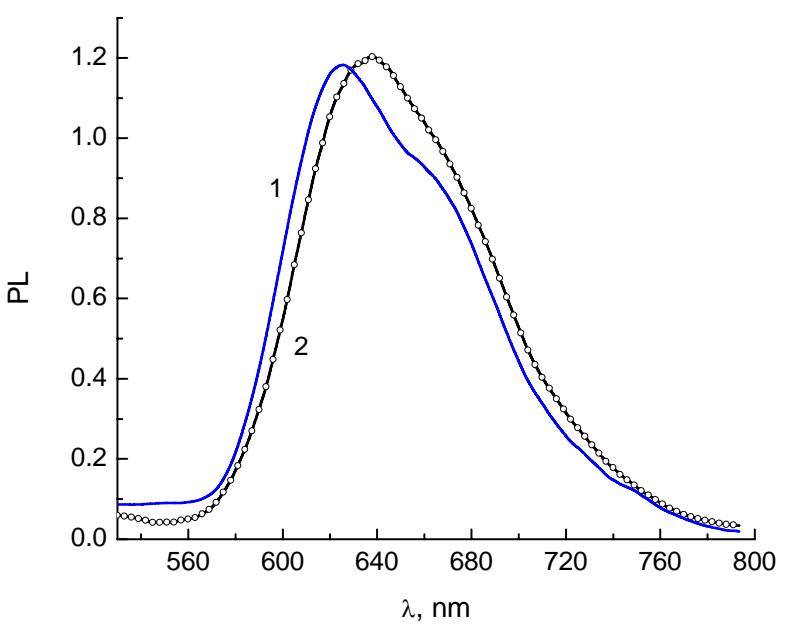

$a$
3.2. Kinetics of photoluminescence of molecular solutions of merocyanine $M-2$

For the molecular solutions studied, the PL lifetimes were measured. The kinetic curves of PL decay of molecular solutions in acetonitrile at $296 \mathrm{~K}$ (a) and $4.2 \mathrm{~K}$ (b) are presented in Fig. 5. At room temperature monoexponential decay of PL with lifetimes in subnanosecond range is observed. At low temperatures ( 77 and $4.2 \mathrm{~K}$ ), the kinetic curves of PL decay of these solutions are well described by the one- and twoexponential function $I_{P L}(t)=A_{1} e^{-t / \tau_{1}}+A_{2} e^{-t / \tau_{2}}$ with the lifetimes of PL in the pico- and nanosecond range. The PL lifetimes determined from the presented exponential dependences of PL decay for different PL wavelengths are summarized in Table 1. The contribution of each of the components of decay in the PL spectrum at the measured wavelength is given in percentages.

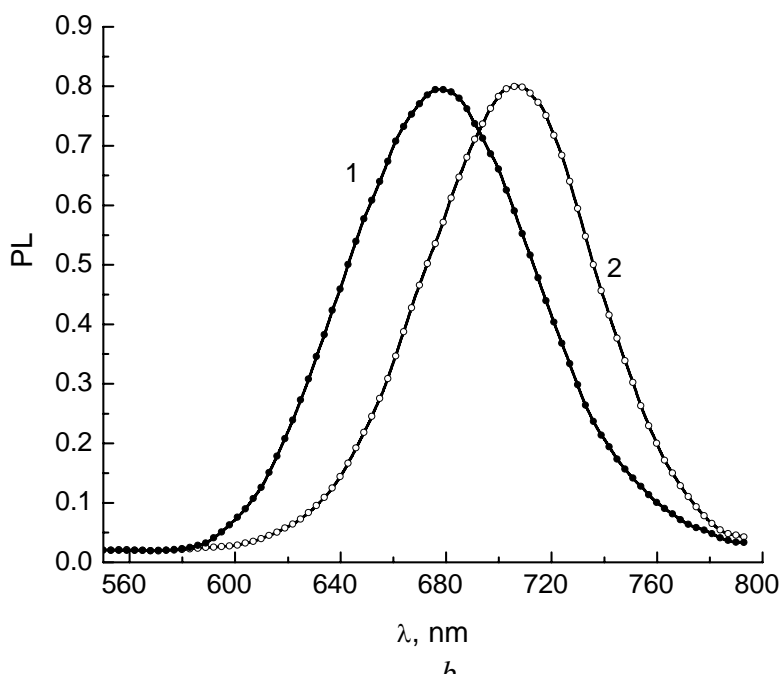

$b$

Fig. 4. The PL spectra of molecular solutions M-2 $(1,2)$ in toluene $(a)$ and acetonitrile $(b)$ at $4.2 \mathrm{~K}(1)$ and $296 \mathrm{~K}(2)$ under excitation $\lambda_{e x}=337.1 \mathrm{~nm}$.
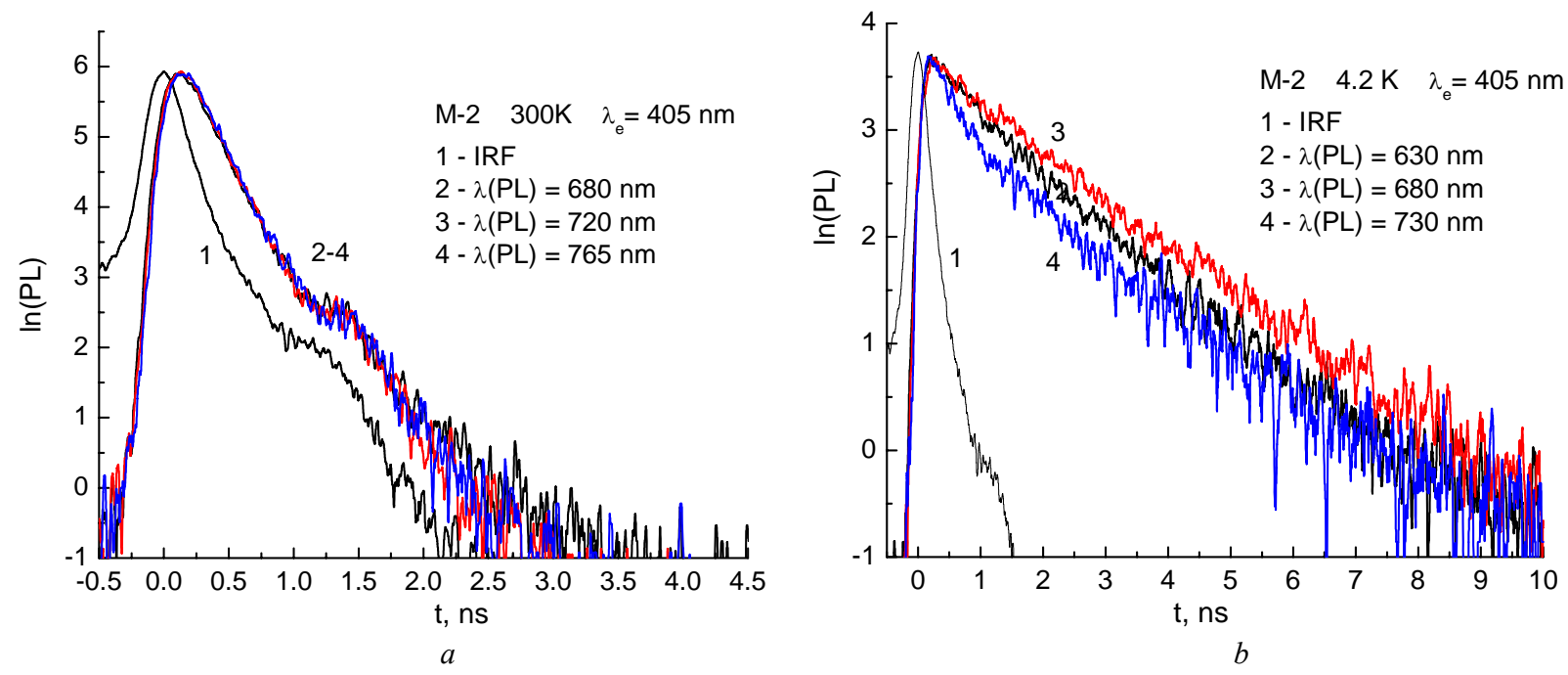

Fig. 5. Kinetic curves of PL decay of molecular solutions M-2 in acetonitrile at $296 \mathrm{~K}(a)$ and $4.2 \mathrm{~K}(b)$. 
As we see from Table 1, the kinetics of PL decay for molecular solutions at room temperature is described by the one-exponential dependence of the PL decay, and the lifetimes are considerably shorter than those at 77 and $4.2 \mathrm{~K}$. At low temperatures, the kinetics of PL decay is described by a two-exponential function with the PL lifetimes $\tau_{1}$ and $\tau_{2}$. Depending on the PL spectral region and temperature of the studied solution, the time $\tau_{1}$ varies from 71 to $268 \mathrm{ps}$, and the time $\tau_{2}$ - within the interval $950 \ldots 2200 \mathrm{ps}$.
3.3. Time-resolved emission PL spectra of molecular solutions of merocyanines

Fig. 6a shows the time-resolved emission spectra (TRES) of M-2 molecular solution in acetonitrile at $300 \mathrm{~K}$. To plot TRES, measurements of the kinetic PL decay curves in the range $0 \ldots 10 \mathrm{~ns}$ for different wavelengths of emission in the spectral region of 570 to $820 \mathrm{~nm}$ with intervals of $2 \mathrm{~nm}$ were performed. According to the given array of kinetic curves for

Table 1. Lifetimes of PL for different wavelengths of the spectrum. The contribution of each component of decay $\tau_{1}$ and $\tau_{2}$ is indicated as a percentage, $\chi^{2}$ is the root-mean deviation of the lifetime.

\begin{tabular}{|l|c|c|c|c|c|}
\hline & $\lambda_{P L}, \mathrm{~nm}$ & $\Delta t_{1 / 2}, \mathrm{ps}$ & $\tau_{1}, \mathrm{ps}$ & $\tau_{2}, \mathrm{ps}$ & $\chi^{2}$ \\
\hline \multirow{3}{*}{$\mathrm{M}-2, T=296 \mathrm{~K}$} & 680 & 100 & 135 & & 1.574 \\
\cline { 2 - 6 } & 765 & 100 & 135 & & 1.680 \\
\hline \multirow{3}{*}{$\mathrm{M}-2, T=77 \mathrm{~K}$} & 630 & 160 & $80(17 \%)$ & $1946(83 \%)$ & 1.597 \\
\cline { 2 - 6 } & 680 & 160 & $86(14 \%)$ & $2200(86 \%)$ & 1.394 \\
\cline { 2 - 6 } & 750 & 160 & $71(42 \%)$ & $950(58 \%)$ & 1.613 \\
\hline \multirow{3}{*}{$\mathrm{M}-2, T=4.2 \mathrm{~K}$} & 630 & 90 & $115(10 \%)$ & $1700(90 \%)$ & 1.184 \\
\cline { 2 - 6 } & 730 & 90 & $129(7 \%)$ & $2096(93 \%)$ & 1.201 \\
\cline { 2 - 6 } & & 90 & $110(20 \%)$ & $1400(80 \%)$ & 1.243 \\
\hline
\end{tabular}

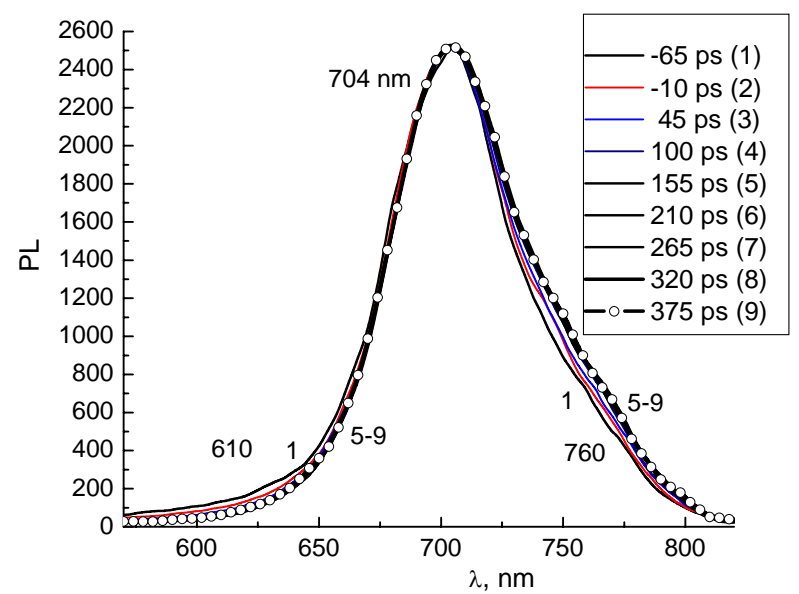

$a$

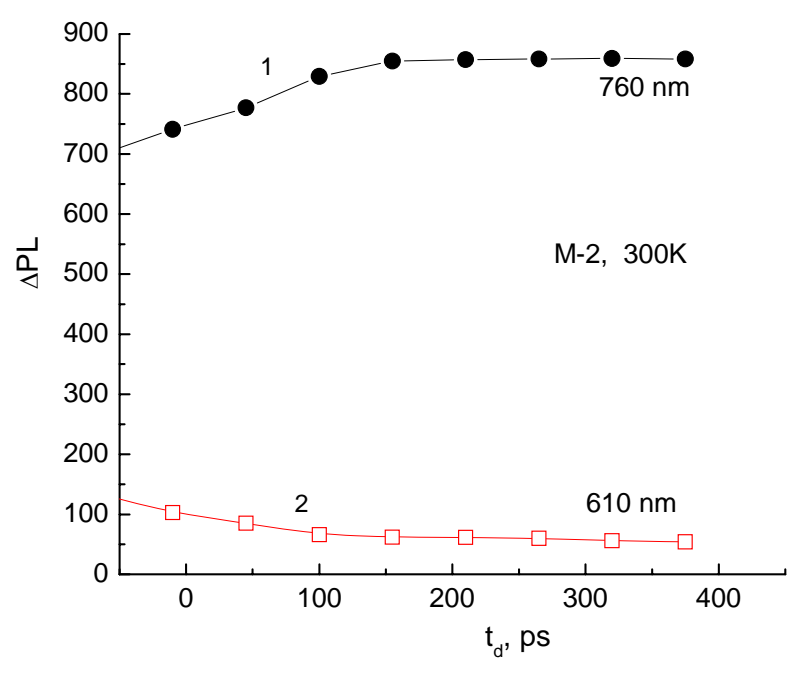

$b$

Fig. 6. (a). Time-resolved emission spectra (TRES) of molecular solutions M-2 in acetonitrile at $300 \mathrm{~K}$. PL was excited by a semiconductor pulsed laser at the wavelength $405 \mathrm{~nm}$. (b) The relative intensity of the bands $760 \mathrm{~nm}(1)$ and $610 \mathrm{~nm}(2)$ for the M-2 solution. 
various delay times $t_{d}$ in the picosecond range, TRES were plotted. The delay time for the constructed instantaneous PL spectra was taken in relation to the maximum of the exciting laser pulse $t_{d}=0$. The presented spectra are normalized to the 706-nm band, for which the lifetime of emission was the longest one. In the PL spectra, three spectral regions can be distinguished: $570 \ldots 650,650 \ldots 710$ and $710 \ldots 800 \mathrm{~nm}$. In the PL spectral region $570 \ldots 630 \mathrm{~nm}$ at the wavelength $610 \mathrm{~nm}$, the relative intensity decreases, and during the time of $100 \mathrm{ps}$ it reaches saturation. In the spectral region of $700 \ldots 800 \mathrm{~nm}$, the relative intensity at the wavelength $760 \mathrm{~nm}$ increases, and for $100 \mathrm{ps}$ the saturation is reached, as can be seen from Fig. 6b for M-2 solution.

Fig. 7 shows the time-resolved emission PL spectra of the M-2 molecular solution in acetonitrile at $4.2 \mathrm{~K}$. The investigated solutions in a silica cuvette with the thickness $4 \mathrm{~mm}$ were placed in helium cryostat. Measurements were made in reflection geometry at a temperature of liquid helium. To plot TRES curves, measurements of the kinetic PL decay curves in the range $0 \ldots 20 \mathrm{~ns}$ for various wavelengths of emission in the spectral region of $550 \ldots 820 \mathrm{~nm}$ at the interval of $2 \mathrm{~nm}$ were performed. Using the given array of kinetic curves for the various delay times $t_{d}$ in pico- and nanosecond ranges, TRES were plotted. The delay time for the plotted instantaneous PL spectra was taken in relation to the maximum of the exciting laser pulse $t_{d}=0$.

Fig. 7 shows TRES for molecular solution M-2 with the delay times $t_{d}=20,110,200-2200$, and 6700 ps. The delay times within the range $200 \ldots 2200$ ps were taken each 200 ps. The presented spectra are normalized to the 685-nm band. As can be seen from Fig. 7, three spectral regions can be distinguished in TRES $570 \ldots 650,650 \ldots 710$, and $710 \ldots 800 \mathrm{~nm}$.

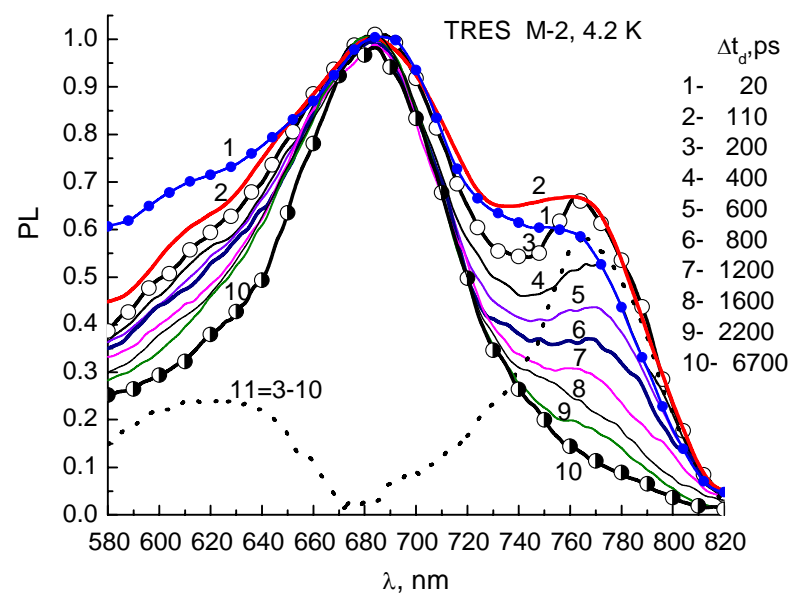

Fig. 7. TRES of molecular solutions $\mathrm{M}-2$ in acetonitrile at the temperature $4.2 \mathrm{~K}$ and under excitation of $405 \mathrm{~nm}$ with the delay times $t_{d}$ of 20 to $6700 \mathrm{ps}$. The spectra are normalized to $685 \mathrm{~nm}$ band.

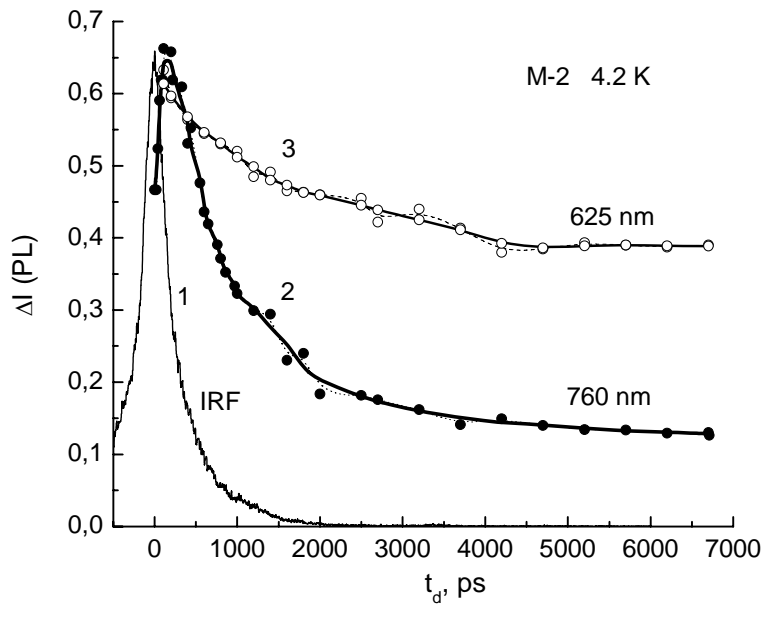

Fig. 8. Change in the intensities of the PL bands of M-2 molecular solutions at the temperature $4.2 \mathrm{~K}$ in the first and third spectral regions relatively to the band $685 \mathrm{~nm} .1-I R F-$ function of system response; $2-\lambda_{P L}=760 \mathrm{~nm} ; 3-\lambda_{P L}=$ $625 \mathrm{~nm}$.

Fig. 8 shows the time dependence of the intensities of PL bands of M-2 solutions (bands 625 and $760 \mathrm{~nm}$ ) in the first and third spectral regions with respect to the band $685 \mathrm{~nm}$. As can be seen from Fig. 8, the relative intensity of the band $625 \mathrm{~nm}$ decreases, when $t_{d}$ changes from 110 to $6700 \mathrm{ps}$. As can be seen from Table 1, the component with $\tau=1980 \ldots 2200 \mathrm{ps}$ gives the main contribution to the PL lifetime in the band $625 \mathrm{~nm}$ at 77 and $4.2 \mathrm{~K}$. In molecular complexes $\mathrm{M}-2$, the relative intensity of the charge transfer band $(760 \mathrm{~nm})$ when changing $t_{d}$ intensively increases in a short time from 0 to $100 \mathrm{ps}$, and then gradually decreases in the time interval from 100 to $6700 \mathrm{ps}$. In this case, the largest changes in the PL intensity are observed, when changing $t_{d}$ in time intervals from 0 to $100 \mathrm{ps}$. Changes in the dynamics of growth and attenuation of the charge transfer band for M-2 merocyanines can be significantly related to conformational changes in fluorophore being in the excited state.

\section{Discussion of results}

\subsection{Resonant structures of researched merocyanines}

Quantum-chemical calculations [9] of the equilibrium molecular geometry of M-2 merocyanines in the ground state indicate that the conjugate part of all molecules is close to planar, which is typical for all the $\pi$-electron systems. Only one of the methylene groups $\left(\mathrm{CH}_{2}\right)$ in the trimethylene bridge comes from the plane of main chromophore. Calculations also showed that double and single bonds are strongly alternate (more than by $0.1 \AA$ ), which is typical for neutral polyene molecules. The length of the formally single bonds $(\mathrm{CH}-\mathrm{CH})$ is approximately equal to $1.45 \AA$, while the lengths of 
formally double bonds $(\mathrm{CH}=\mathrm{CH})$ vary around the values $1.35-1.36 \AA$. In contrast, the lengths of the bonds in the benzene ring is practically aligned and equals $1.40 \pm 0.03 \AA$.

In the theoretical model $[3,5]$, merocyanines are considered as conjugated $\mathrm{D}-\mathrm{A}$ systems with the following structure:

\section{$\mathrm{D}-(\mathrm{CH}=\mathrm{CH}) \mathrm{n}-\mathrm{A}$ or $\mathrm{D}-\pi-\mathrm{A}$.}

In our case, the donor fragment contains atom of nitrogen with a lone pair of electrons, that can be differently conjugated to main chromophore in the ground or excited state. The acceptor fragment is $\mathrm{C}(\mathrm{CN})_{2}$ group. The polyene chain with its $\pi$-electronic system acts as a bridge between the donor and acceptor groups. To provide correct interpretation of the conjugated $\mathrm{D}-\pi-\mathrm{A}$ complexes, a description from the viewpoint of resonance between the forms $\mathrm{A}$ and $\mathrm{B}$ is used, as it is shown in Fig. 9. Here, the structure A corresponds to the neutral form of an ideal polyene state with maximum alteration of the orders of simple and double bonds and zero charges on carbon atoms. The structure B corresponds to the bipolar form of ideal polyene with a maximum separation of unlike charges and alternation of the order of simple and double bonds. Depending on the donor and acceptor forces of the finite groups as well as on polarity of the solvent, one of the merocyanine structures presented in Fig. 9 can be realized. Theoretically, equilibrium in the system of two A and B forms is achieved in the so-called "cyanine-like state" $\mathrm{C}$. The structure $\mathrm{C}$ corresponds to the ideal polymethine (cyanine) state with the aligned (semi-quary) orders of simple and double bonds and alternation of partially positive and negative charges. An increase in polarity of the solvent and transition of molecule to the excited state promotes equalization of the orders of single and double bonds, alternation of charges, and tranfer to a structure close to $\mathrm{C}$.
4.2. Possible types of charge transfer processes and ways of relaxation in excitation state

Possible types of processes of charge transfer in the excited state for D-A systems are discussed in [10]. The wave function $\Psi$ of the excited state of molecules with intramolecular charge transfer can be represented as a superposition of the neutral (local) excited $\Phi_{f}(\mathrm{D}-\pi-\mathrm{A})^{*}$, with charge transfer $\Phi_{I}\left(\mathrm{D}^{+}-\pi-\mathrm{A}^{-}\right)$and a set of molecular vibrational $\chi_{i}$ states $[10,11]$ :

$\Psi=\mathrm{C}_{1} \Phi_{f}+\mathrm{C}_{2} \Phi_{I}+\sum \mathrm{C}_{i} \chi_{i}$

Similarity of the PL excitation spectra and absorption spectra, independence of the position of absorption spectra from solvent polarity and independence of the form of stationary PL spectra from the wavelength of excitation in a wide spectral region indicate that, at the initial moment of time, after optical excitation in the absorption band, the molecules of the studied merocyanines with high probability are in the $\Phi_{f}(\mathrm{D}-\pi-\mathrm{A})^{*}$ excited Frank-Condon state. Only with time the luminescent state $\Phi_{I}$ may occur, which is confirmed by a significant Stokes shift of the PL spectra. The last term of expression (1) characterizes the competing channel of intramolecular relaxation, specific weight of which is the quantum yield of intramolecular ionization $\left(\mathrm{D}^{+}-\pi-\mathrm{A}^{-}\right)$.

Suppose that the molecular system at the time $t=0$ is in locally excited state with the wave function $\Phi_{f}$. Over time, the state $\Phi_{I}$ arises and its probability of density reaches its maximum value

$$
P_{m}=\frac{4 \beta^{2}}{4 \beta^{2}+(\Delta E)^{2}}=\frac{1}{1+\frac{(\Delta E)^{2}}{4 \beta^{2}}}
$$

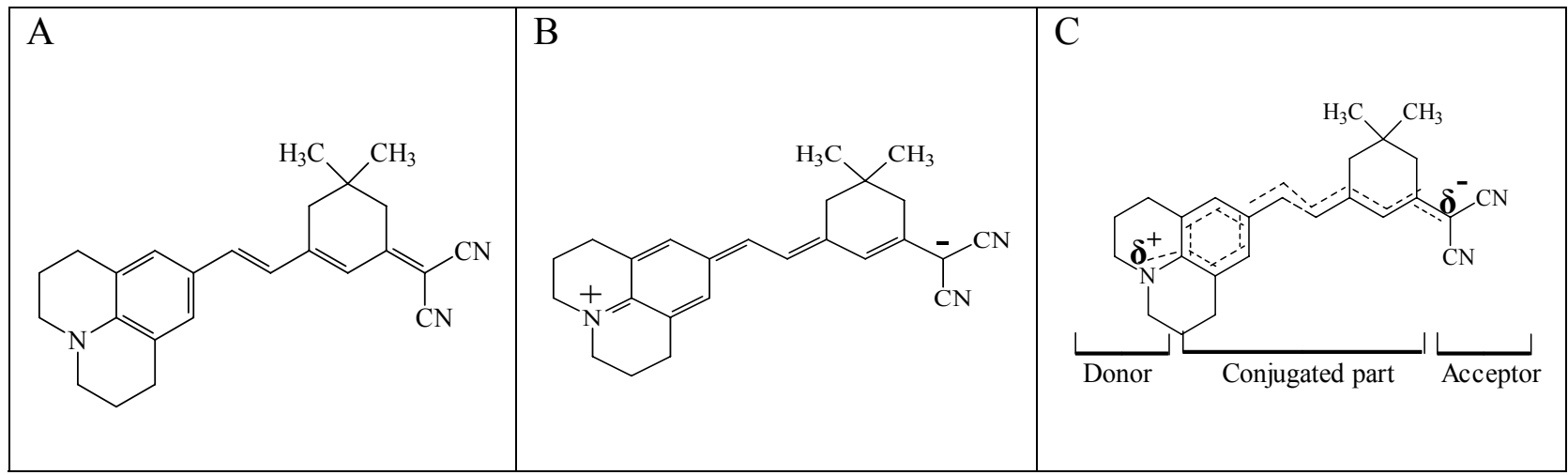

Fig. 9. Resonant structures of merocyanine M-2: A - neutral polyene; B - bipolar polyene; C - ideal polymethine. 
at the time

$$
t_{m}=\frac{h}{4 \beta\left[1+\frac{(\Delta E)^{2}}{4 \beta^{2}}\right]^{1 / 2}},
$$

where $\beta=\left\langle\Phi_{f}\left|\mathrm{H}^{\prime}\right| \Phi_{I}\right\rangle$ is the matrix element of electron interaction between states with the wave functions $\Phi_{f}$ and $\Phi_{I}$, and $\Delta E$ - energy difference between the corresponding configurations. The transition speed $f$ between the states with the wave function $\Phi_{f}$ and $\Phi_{I}$ can be defined as the ratio of the maximum probability density $P_{m}$ to $t_{m}$ :

$$
f=\frac{4 \beta}{h\left[1+\frac{(\Delta E)^{2}}{4 \beta^{2}}\right]^{1 / 2}}
$$

The transition rate $f$ represents the frequency with which electron oscillates between the molecule parts D and $\mathrm{A}$. In the case of resonance, we have $f=4 \beta / h$. If intramolecular charge transfer occurs much faster than the period of molecular oscillations, then the electronic state of the molecule can be represented by the wave function of the form (1), and this case describes strong interaction. By its nature, the case of strong interaction corresponds to the excitation of exciplexes. If the charge transfer rate is comparable or less than vibrational relaxation, then the state with the wave function $\Phi_{I}$ is realized, and we deal with the case of weak interaction. Exactly this classification was proposed by Förster [12] for the intermolecular transfer of electron excitation.

In the limit case of very weak interaction, one can enter the usual probability of transition, defining it as

$W=\frac{2 \pi}{\hbar}|\beta|^{2} \rho_{f}$

where $\rho_{f}$ is the level of density in the state $\Phi_{f}$. After the completion of the vibrational relaxation following the charge transfer, the state of charge transfer can be further stabilized as a result of reorientation of the surrounding molecules in polar solvent.

Spectral properties of many of merocyanines in the excited state are close to the properties of symmetric cyanines [4-6], where the so-called "cyanine limit" states can be realized, that is, a state with maximally aligned lengths of bonds is achieved.

We can interpret the first electronic transition $\mathrm{S}_{1}$ in merocyanines M-2 (absorption band $545 \mathrm{~nm}$ ) as cyanine-like (or cyanine) tranfers. In addition, these transitions are polarized along the polymethine chromophore [9], similar to the tranfers in cyanine dyes.
Table 2. Calculated characteristics of the molecule (ZINDO/S method, AM1-geometry).

\begin{tabular}{|c|c|c|c|c|c|}
\hline Molecule & Transfer & $\lambda, \mathrm{nm}$ & $\mu_{i}, \mathrm{D}$ & $\Delta \mu_{i}, \mathrm{D}$ & $\begin{array}{c}\mu_{0} \rightarrow i, \\
\mathrm{D}\end{array}$ \\
\hline $\mathrm{M}-2$ & $\begin{array}{c}\mathrm{S}_{0} \\
\mathrm{~S}_{0} \rightarrow \mathrm{S}_{1}\end{array}$ & 540 & $\begin{array}{c}11.245 \\
19.120\end{array}$ & 7.875 & 9.820 \\
\hline
\end{tabular}

Table 2 shows calculations [9] of dipole moments $\mu_{i}$ in the ground $S_{0}$ and excited $S_{1}$ states, the energy of these transitions and dipole moments $\mu_{0 \rightarrow i}$ of these electronic transitions. We see that, also as asymmetric linear conjugated molecules, the molecules of the studied merocyanines have a significant dipole moment in the ground state that varies considerably when being excited.

Significant redistribution of electronic density inside chromophore at $\mathrm{S}_{0} \rightarrow \mathrm{S}_{1}$ transition is the reason for further significant changes in the occupancy of bonds or changes in the orders of bonds $p_{\mu v}$. Initial relaxation that follows the change in the occupancy of the bonds leads to the excited state $S_{1}\left(R_{1}\right)$ (Fig. 10) with significant alignment of the lengths of bonds in chromophore. This alignment of the bonds, as it was noted above, is typical for cyanine dyes [13], and we can treat the state $S_{1}\left(R_{1}\right)$ and corresponding relaxation as cyanine-like, too.

As can be seen from Fig. 5, after the exciting laser pulse, there is observed some delay in the appearance of emission. This delay may be interpreted as the time for a radiationless transition from $S_{1}$ to $S_{1}\left(R_{1}\right)$ and establishment of equilibrium state there. This delay $\Delta t_{1 / 2}$ determined from relation to the front of the laser pulse at its half-width is presented in Table 1. As can be seen from Table 1, for merocyanines $\mathrm{M}-2$ in the range of temperatures of 300,77 and $4.2 \mathrm{~K}$, this delay varies from 90 up to $150 \mathrm{ps}$.

The emission spectra of the excited state $S_{1}\left(R_{1}\right)$ for the M-2 merocyanines are shown in Fig. 4. From Fig. 4b it is seen that the maxima of the PL spectra are significantly shifted to the long-wave region relative to the absorption, indicating significant changes in equilibrium energy under relaxation in the excited state. This is in good agreement with the significant difference in molecular geometry (especially in differences in the lengths of bonds) of the ground $\mathrm{S}_{0}$ and cyanine-like relaxed $\mathrm{S}_{1}\left(\mathrm{R}_{1}\right)$ states. It is shown in [14] that the Stokes shift $\Delta v_{s}=v_{\max }(\mathrm{abs})-v_{\max }(\mathrm{PL})$ directly depends on the change in the lengths of the bonds in ground $\mathrm{R}_{\mu \nu}^{0}$ and excited $\mathrm{R}_{\mu \nu}{ }_{\mu \nu}$ states. Here, the indexes $\mu$ and $v$ run through all the $\pi$-bonds. Between the length $\mathrm{R}_{\mu \nu}$ and order $\mathrm{p}_{\mu \nu}$ of $\mathrm{C}-\mathrm{C}$ bonds in the conjugate system the interconnection is well-known [15]:

$\mathrm{R}_{\mu v}(\AA)=1.58-0.13 \mathrm{p}_{\mu \nu}$. 

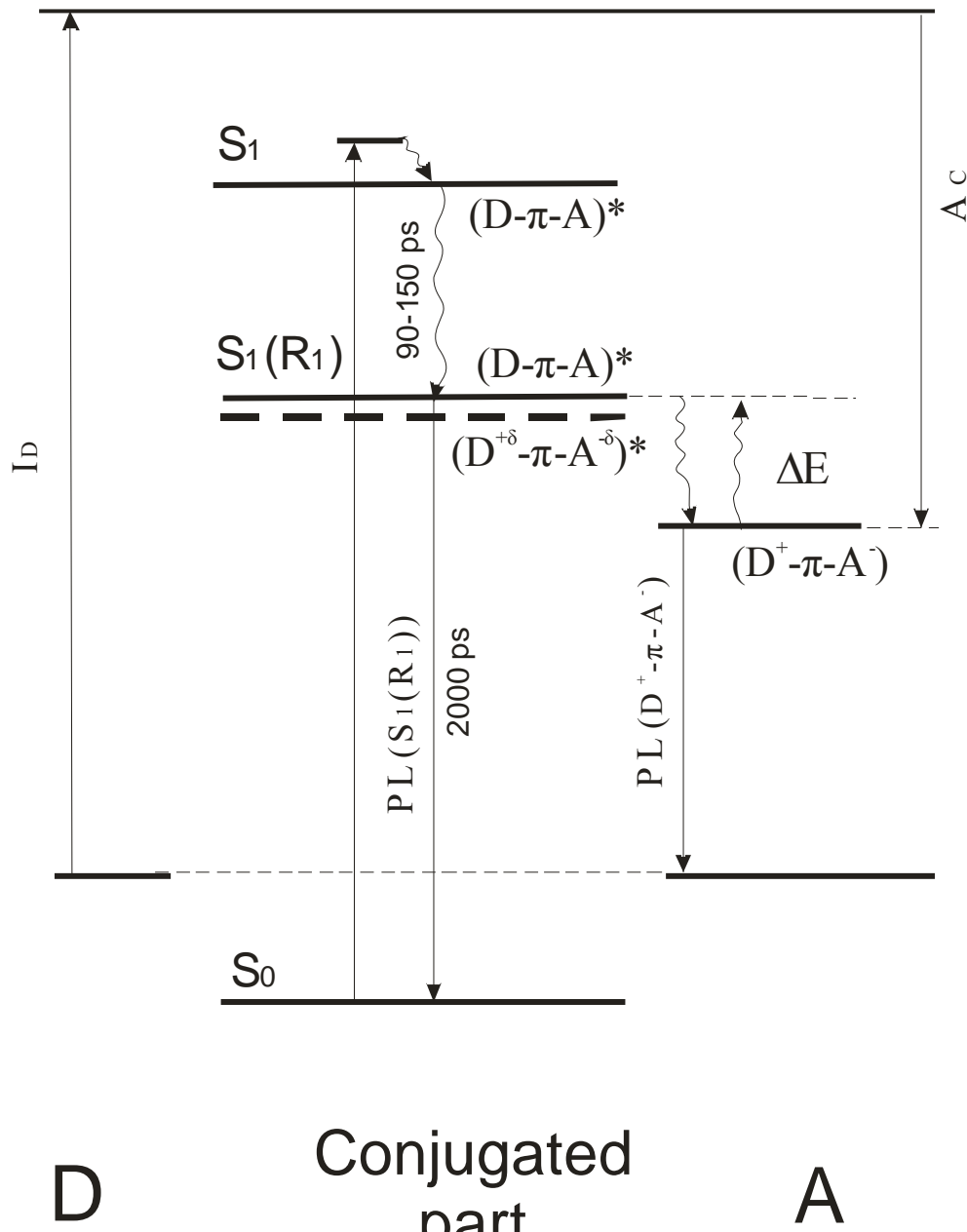

\section{Conjugated part}

Fig. 10. Schematic arrangement of orbitals in the M-2 molecule.

We can assume that this ratio can be used to approximate the relaxation of geometry of the molecule after excitation. Taking into account the correlation between the values of $\mathrm{R}_{\mu v}$ and $\mathrm{p}_{\mu \nu}$, as previously proposed in [16], it is possible to present changes in the orders of bonds by the relation:

$$
\delta\left(\mathrm{p}_{\mu \nu}\right)=\sqrt{\sum\left(\mathrm{p}_{\mu \nu}^{*}-\mathrm{p}_{\mu \nu}^{0}\right)^{2}}
$$

The magnitude of $\delta\left(\mathrm{p}_{\mu v}\right)$ can be considered as an integral characteristic of possible geometric changes under relaxation in the excited state (taking into account only changes in the lengths of bonds in chromophore, which provides the planarity of the molecule, and PL that occurs here). We can assume that the parameter $\delta\left(\mathrm{p}_{\mu v}\right)$ will correlate with the Stokes shift. With the bonds in the open chain and in the benzene ring, for the M-2 molecule we calculated $\delta\left(\mathrm{p}_{\mu v}\right)=0.896$ [9]. This value exceeds the value for similar values for symmetric cyanine dyes: $\delta\left(\mathrm{p}_{\mu v}\right)=0.120 \ldots 0.180$. Stokes shifts in the spectra of merocyanine $\left(2793 \mathrm{~cm}^{-1}\right.$ in toluene and $4185 \mathrm{~cm}^{-1}$ in acetonitrile for M-2) are higher than in the spectra of symmetric cyanines: $\Delta v_{s}=400 \ldots 700 \mathrm{~cm}^{-1}[17]$.

The effects of solvation influenced significantly on the conformational state of merocyanines in the polar solvent, especially in the excited state. This means that in the excited state the molecules are planar. Fig. $4 \mathrm{~b}$ also shows that at low temperatures in the PL spectra, more complex bands are observed as compared to the bands at room temperatures. First of all, as it was supposed, the maxima of PL bands in the frozen solution, when there is no reorientation of the dye and solvent, are displaced gipsochromously. But the main characteristic of the spectra at low temperature is related to the shape of the spectral bands. The width of the PL bands at $4.2 \mathrm{~K}$ significantly exceeds the band width at high temperatures. The half-width of the PL band at room temperature equals $1890 \mathrm{~cm}^{-1}$, and at $4.2 \mathrm{~K}-2015 \mathrm{~cm}^{-1}$. This phenomenon contradicts the well-known experimental and substantiated theoretically data, where the PL band width usually narrows at low temperatures, since the intensity of the vibrational interactions is reduced [18]. As a result based on the considerations above, we can assume that such transformations of 
spectral bands, especially their expansion, are related to the existence of additional components that are formed due to conformational changes in the polymer chain under relaxation of the excited state.

In TRES of molecular solutions, we conditionally distinguished three spectral regions, where the structure of the spectra is different (Fig. 7). The presence of the first spectral region we related with the fact that in solutions, the presence of some part of the molecules with incomplete conjugation of the $\mathrm{D}$ dimethylamine fragment with benzene and polymethine fluorophore are possible. For the majority of M-2 molecules in the excited state, complete conjugation of molecular fluorophore is revealed, and the main PL belongs to the second spectral region - the band $685 \mathrm{~nm}$, which corresponds to the ground relaxed state $\mathrm{S}_{1}\left(\mathrm{R}_{1}\right)$. The third region corresponds to $\mathrm{PL}$ from the states with charge transfer $\left(D^{+}-\pi-A^{-}\right)$- the band $760 \mathrm{~nm}$.

To transfer the charge and localize it on the acceptor fragment, it is necessary that the charge transfer rate is comparable or less than vibrational relaxation. In this case, the state with the wave function $\Phi_{I}$ is realized, and we deal with the case of weak interaction. This case of charge transfer can be compared with the mechanism for the intermolecular transfer of electron excitation, which was proposed by Förster [12]. If the intramolecular charge transfer occurs much faster than the period of molecular vibrations, then the electronic state of the molecule can be represented by the wave function of the form (1) and this case describes a strong interaction. This case is realized for fluorescence states $S_{1}\left(R_{1}\right)$. By its nature, this is a case of strong interaction, and it can be compared with an excitation of exciplexes. In the case of the classical exciplex $[19,20]$, the resonant interaction and with charge transfer interaction between the D and molecules of a certain stoichiometric composition appear when in the excited state they are located at the interplanar distance of $0.30 \ldots 0.36 \mathrm{~nm}$. In our case, the resonant interaction and interaction with charge transfer between the D and A molecules occurs through a conjugated carbon chain.

As can be seen from Table 1, these states are polar and are realized as a result of resonant and charge transfer interaction. The lifetime of this state is close to 2000 ps. The presence of states with charge transfer can be investigated based on two-photon absorption and two-photon excited photoluminescence. For excitation of two-photon PL, the base radiation of the Nd:YAG laser was used $\left(\lambda_{e x}=1064 \mathrm{~nm}, t=10 \mathrm{~ns}, N=0.05 \ldots 1.0 \mathrm{MW}\right.$, $f=50 \mathrm{~Hz}$ ), and for the one-photon PL - the second harmonic of the Nd:YAG laser $\left(\lambda_{e x}=532 \mathrm{~nm}\right.$, $N=0.1 \ldots 0.5 \mathrm{MW}, f=50 \mathrm{~Hz})$. Registration of onephoton PL was carried out by using a stroboscopic system $[21,22]$ that allowed recording the kinetics and PL spectra with a different time delay $t_{d}$ in relation to the laser pulse. As a spectral device, a monochromator MDR-12 was used. Registration of the spectra of oneand two-photon-excited PL was carried out at the maximum of laser pulse with a time window of $100 \mathrm{ps}$.
The intensity of PL of solutions M-2 in acetonitrile under excitation of them with the base $\left(\lambda_{e x}=1064 \mathrm{~nm}\right)$ harmonic of the Nd:YAG laser was quadratic. Fig. 11 shows the PL spectra of M-2 solutions in acetonitrile under one-photon (curve 1) and two-photon (curve 2) excitation. The spectra are normalized to the maximum of PL bands. In the PL spectra of the solutions studied, there is a slight long-wave shift of the bands of twophoton excited PL in relation to the one-photon excited PL. For a one-photon excited PL, the maxima of emission bands are observed at $709 \mathrm{~nm}$, and for the twophoton excited PL - at $714 \mathrm{~nm}$.

One- and two-photon transfers are subject to different selection rules and provide additional, with respect to each other, spectroscopic information. The probability $W$ of absorbance of two photons with the frequency $\omega$ and corresponding polarization of electric field in the direction $\mathbf{e}$ in a centrosymmetric environment in the dipole approximation has the form $[22,23]$ :

$$
\begin{aligned}
& W(\omega, \mathbf{e}) \propto N \omega^{2} I^{2} \sum_{f}\left|M_{f g}\right|^{2} \delta\left(2 \omega-\omega_{f g}\right), \\
& M_{f g}=\sum_{i} \frac{2 p_{f i} p_{i g}}{\omega-\omega_{i g}},
\end{aligned}
$$

where $g, i, f$ denote the ground, intermediate and final states of the two-photon transfer; $M_{f g}$ is the composite matrix element of transfer between the states $g$ and $f$; $p_{i j}$ - matrix element of the dipole moment of transfer (projection onto direction e) between different $i$ and $j$ states; $\hbar \omega_{i j}=E_{i}-E_{j}$ - energy difference between the states $i$ and $j ; I-$ intensity of the exciting light; $N-$ density of molecules in the environment. It is usually assumed that the main contribution to the probability of two-photon transfer is made by those intermediate states $i$ that are close to the levels $g$ and $f$.

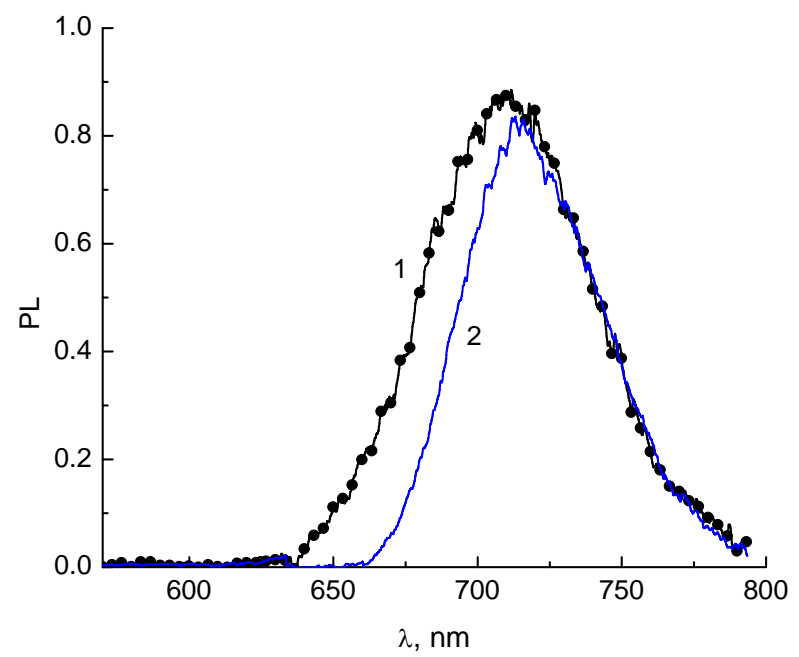

Fig. 11. PL spectra of M-2 molecular solutions in acetonitrile at $295 \mathrm{~K}$ and delay times $t_{d}=0$ under excitation at the wavelengths $\lambda_{e x}=532 \mathrm{~nm}(1)$ and $1064 \mathrm{~nm}$ (2). 
In an environment with a center of symmetry, the ground state is non-degenerate and the electronic states can be separated into even $(g)$ and odd $(u)$. In the dipole approximation, one-photon transfers from $(g)$ into $(g)$ or from $(u)$ to $(u)$ states are forbidden, but two-photon transitions between these states are allowed. Thus, when using two-photon absorption spectroscopy, one can probe new electronic states that cannot be observed at one-photon absorption. These states also include states with charge transfer (CT-states) forbidden at one-photon absorption.

If the system loses inversion symmetry and has static dipole moment, then the ground state is degenerate and has no certain symmetry [24]. In this case, there is no strict forbiddance for the transfers between the ground and excited states at one- and two-photon absorption of light. Expression (2) for a molecular system without center of symmetry can be rewritten by separating the static $\mu_{i i}$ and transition $p_{i j}$ dipole moments $[25,26]$ :

$M_{f g}=\sum_{i} \frac{2 p_{f i} p_{i g}}{\omega-\omega_{i g}}-2 \frac{\Delta \mu_{f g} p_{f g}}{\omega}$

where $\Delta \mu_{f g}=\mu_{f f}-\mu_{g g}$ is the difference static dipole moment (projection on the direction e) between the final and ground states. The intensity of the two-photon excited PL is equal to

$I_{P L}=\eta \alpha(2 \omega) I^{2}$,

where $\eta$ is the quantum yield of PL, $\alpha(2 \omega)$ - two-photon absorption coefficient. The absorption coefficients for one- and two-photon absorption for a two-level system without a center of inversion can be represented as

$$
\begin{aligned}
& \alpha(\omega) \propto p_{f g}^{2} \delta\left(\omega-\omega_{f g}\right), \\
& \alpha(\omega) \propto p_{f g}^{2} \Delta \mu_{f g}^{2} \frac{F(\omega)}{\hbar \omega} \delta\left(\omega-\omega_{f g}\right) .
\end{aligned}
$$

\subsection{Soliton mechanism of charge transfer in merocya- nine $M-2$}

Theoretically, the process of electron transfer in molecular $\mathrm{D}-\pi-\mathrm{A}$ complexes, similar to $\mathrm{M}-2$, within the frame of one-dimensional model was considered in $[1$, $27,28]$. The rate of electron transfer in this $\mathrm{D}-\pi-\mathrm{A}$ complex essentially depends on the conformational state of the polymer chain. As polymer, i.e., the main part of the molecular complex, a carbon chain has been taken, it contains two mesomeric conformations - homogeneous (similar to that in Fig. 9C) and dimerized (similar to that in Fig. 9A).

In the first case, the carbon atoms are located equidistantly, and we have a collectivized electronic density ( $\pi$-conjugation). In the second case, there is alternation of bonds, that is, dimerization. Dimerization in the chain is manifested in such a way that the $\pi$ conjugation for the whole chain is absent, and in addition to acoustic phonons, optical phonons appear in this system.

Thus, in the first case, the electron is completely delocalized in a one-dimensional chain, and the electron transfer follows a soliton type [27, 28]. Carbon chains of this type are a good transport environment. In the second case, deformation of the chain in the system leads to a decrease in the symmetry (dimerization) of the chain and the appearance of a new oscillatory frequency that corresponds to local oscillations of the double bond. These vibrations provide localization of electron in each node (the polaron effect), thereby reducing total delocalization in the system, which takes place in the first case when there is a collective vibrational packet (coherent state). As a result, in the second case, the probability to find electron in A is greatly reduced.

From the above discussion, one can analyze the kinetics of PL associated with the states of charge transfer. Presented in Fig. 8 initial sharp increase in the intensity of the PL band at $760 \mathrm{~nm}$ can be related with the soliton mechanism of transfer of charge density through the relaxed polymethine chain in its homogeneous coherent state in time $t_{d}=100 \mathrm{ps}$. It is approximately equal to the propagation rate of electron excitation close to $1.45 \cdot 10^{3} \mathrm{~cm} \cdot \mathrm{s}^{-1}$. This value is essentially less than the sound speed. In the dimerized case, with the same value of the constant of electronvibrational interaction, there is only a partial delocalization of electron, which significantly degrades the transfer properties of the system and increases the time of transfer of electron along the polymeric chain, which in our case is equal to $950 \ldots 1400$ ps.

\section{Conclusions}

1. Absorption of molecular polar acetonitrile solutions M-2 (band $545 \mathrm{~nm}$ ) is the Frank-Condon transfer from $\mathrm{S}_{0}$ to $\mathrm{S}_{1}$ state. This transition is characterized by a change in the distribution of electronic density in the polymer carbon chain without changing in the orders of the bonds. The distribution of electronic density in a chain is characteristic for polymethine (cyanine) and is characterized by alternation of negative and positive charges.

2. The process of relaxation from $S_{1}$ to $S_{1}\left(R_{1}\right)$ state goes with the alignment of the lengths of bonds for the time close to $90 \ldots 150 \mathrm{ps}$ at $4.2 \mathrm{~K}$. This manifests itself in the PL spectra by substantial Stokes shift of the bands. Emission transfer from the state $\mathrm{S}_{1}\left(\mathrm{R}_{1}\right)$ (band $680 \mathrm{~nm}$ ) is characteristic for exciplex PL. The lifetime of PL in the $680 \mathrm{~nm}$ band at $4.2 \mathrm{~K}$ is close to $2000 \mathrm{ps}$.

3. The PL band $(760 \mathrm{~nm})$ of the state with charge transfer for the molecular $\mathrm{D}-\pi-\mathrm{A}$ complex studied is better manifested in time-resolved emission PL spectra at low temperatures. The kinetics of PL decay in the 
$760 \mathrm{~nm}$ band at low temperatures ( 77 and $4.2 \mathrm{~K}$ ) is described by the two-exponential function with the PL lifetimes $\tau_{1}=71 \ldots 110 \mathrm{ps}$ and $\tau_{1}=950 \ldots 1400 \mathrm{ps}$. Two lifetimes of PL in the band with charge transfer can be related to two mechanisms of charge transfer in the process of radiative recombination: a coherent (soliton), characteristic for the polymethine conformation of carbon chain, and polaron, characteristic for the polyene conformation of the carbon chain.

\section{References}

1. Davydov A.S. Solitons in quasi-one-dimensional molecular structures. Uspekhi fizich. nauk. 1982. 138, No. 4. P. 603-643 (in Russian).

2. Suponitsky K.Yu., Timofeeva T.V., Antipin M.Yu. Molecular and crystal design of nonlinear optical organic materials. Russ. Chem. Rev. 2006. 75, No. 6. P. 457-498.

3. Meyers F., Marder S.R., Perry J.W. Introduction to the nonlinear optical properties of organic materials, in: Chemistry of Advanced Materials: an Overview, L.V. Interrante and M.J. HampdenSmith (eds.). Wiley, New York, 1998. P. 207-269.

4. Kulinich A.V., Ishchenko A.A. Merocyanine dyes: Synthesis, structure, properties and applications. Russ. Chem. Rev. 2009. 78, No. 2. P. 141-145.

5. Daehne S. Color and constitution: One hundred years of research. Science. 1978. 199. P. 1163 1167.

6. Meyers F., Marder S.R., Perry J.W., Bredas J.L. Electric field modulated nonlinear optical properties of donor-acceptor polyenes: Sum-overstates investigation of the relationship between molecular polarizabilities $(\alpha, \beta$ and $\gamma$ ) and bond length alternation. J. Amer. Chem. Soc. 1994. 116. P. 10703-10714.

7. Lemke R. Solvatochromie von $80 \mu \mathrm{m}$ in verschiedenen Alkoholen bei ArylidenisophoronAbkömmlingen. Chem. Ber. 1970. 103. P. 1894 1899.

8. Lemke R. Knoevenagel condensation in dimethylformamide. Synthesis. 1974. 5. P. 359-361.

9. Sevryukova M.M., Piryatinski Yu.P., Vasylyuk S.V., Yashchuk V.M., Viniychuk O.O., Gerasov A.O., Slominskii Yu.L., Kachkovsky O.D. Cyanine-like and polyenic relaxation paths of merocyanine derivatives of malonodinitrile in the excited state detecting by low temperature timeresolved fluorescence. Ukr. J. Phys. 2012. 57, No. 8. P. 812-823.

10. Mataga N. Properties of molecular complexes in the excited electron states, in: Molecular Interactions, Ed. by H. Ratajczak and W.J. OrvilleThomas, Vol. 2. Wiley, New York, 1980.

11. Silinsh E.A., Kurik M.V., Chapek V. Electron Processes in Organic Molecular Crystals.
Phenomena of Localization and Polarization. Riga, Zinatne, 1988 (in Russian).

12. Forster T. Delocalized excitation and excitation transfer. In: Modern Quantum Chemistry, O. Sinaoglu (ed.), Vol. 3. Academic Press, New York, 1965. P. 93-137.

13. Dewar M.J.S. The Molecular Orbital Theory of Organic Chemistry. McGraw-Hill, New York, 1969.

14. Hunt G.R., McCoy E.F., Ross I.J. Excited states of aromatic hydrocarbons: Pathways of internal conversion. Austral. J. Chem. 1962. 15, No. 4. P. 591-604.

15. Ishchenko A.A. Laser media based on polymethine dyes. Quantum Electronics. 1994. 24, No. 6. P. 471-493.

16. Kachkovskii A.D. The nature of electronic transitions in linear conjugated systems. Russ. Chem. Rev. 1997. 66, No. 8. P. 647-664.

17. Lacowicz J.R. Principles of Fluorescence Spectroscopy. Plenum, New York, 1983.

18. Chemla D.S. and Zyss J. (Eds.) Nonlinear Optical Properties of Organic Molecules and Crystals. Academic Press, Inc., 1987. 1. 385 p.

19. Leonhardt H., Weller A. Elektronenübertragungs reaktionen des angeregten Perylens. Ber. BunsenGes. Phys. Chem. 1963. 67. P. 791-795.

20. Walker M., Bodnar T., Lumry R., Exciplex formation in the excited state of indole. J. Chem. Phys. 1966. 45, No. 9. P. 3455-3457.

21. Piryatinski Yu., Yaroshchuk O. Photoluminescence of $n$-pentyl- $n$-cyanobiphenyl in liquid crystalline and solid crystal state. Optics and Spectroscopy. 2000. 89, No. 6. P. 937-943 (in Russian).

22. Shen Y.R. The Principles of Nonlinear Optics. New York, John Wiley \& Sons, Inc., 1984.

23. Bredikhin V.I., Galanin M.D., Genkin V.N. Twophoton absorption and spectroscopy. Uspekhi fizich. nauk. 1973. 110. P. 1-43 (in Russian).

24. Pantell P.H., Puthoff H.E. Fundamentals of Quantum Electronics. New York, John Wiley \& Sons, Inc., 1969.

25. Piryatinski A., Deck R. Excimer states and enhanced two-photon absorption in intramolecular charge-transfer crystals. Chem. Phys. Lett. 1997. 269. P. 156-160.

26. Abe S. Two-photon probe of forbidden exciton states in symmetric aggregates of asymmetric molecules. Chem. Phys. 2001. 264. P. 355-363.

27. Yaltychenko O.V., Kanarovskii Yu. The electron transfer in the donor-polymer-acceptor molecular complex. Surf. Eng. and Appl. Electrochem. 2008. 44, No. 6. P. 477-479.

28. Lutsyk P., Piryatinski Yu., Kachkovsky O., Verbitsky A., and Rozhin A. Unsymmetrical relaxation paths of the excited states in cyanine dyes detected by time-resolved fluorescence: Polymethinic and polyenic forms. J. Phys. Chem. A. 2017. 121, No. 43. P. 8236-8246. 